\title{
Open Access Networks
}

\author{
Einar Edvardsen, Thor Gunnar Eskedal, André Årnes
}

Telenor Research and Development, Norway.

Abstract: This paper introduces an innovative approach to an Open Access Network (OAN) built upon the existing "privately owned" wireless LANs and the users' access lines (ADSL/VDSL, optical fibre, cable modems). By providing up to $25 \mathrm{Mb} / \mathrm{s}$ shared capacity to the stationary and casually visiting users in a wireless LAN zone, the OAN is probably the only realistic alternative to realize the future $3 \mathrm{G}$ and $4 \mathrm{G}$ visions for mobile communication. An OAN has the potential to integrate P2P communication into legacy networks, thus new market segments will be opened for network and service providers, and new and improved services can be offered to the customers. The paper contains a superficial analysis of the actual elements needed to realise the network. Also, some of the most important network behaviour and performance parameters are identified and discussed.

Key words: OAN, Open Access Network, wireless LAN, DSL, broadband

\section{INTRODUCTION AND RATIONALE}

The establishment of broadband networks is progressing rapidly in the industrialised parts of the world. Telecom operators are offering broadband access to the public by installing DSL technology on their telephone lines, cable modems on their TV cables and optical fibres. In a few years, these existing access networks will be upgraded to provide real broadband access distributed far out in the network.

Choosing the example of ADSL, these kinds of modems are able to transfer up to $8 \mathrm{Mb} / \mathrm{s}$ to each customer. Practical installations of such modems, however, perform less, but compared to yesterday's telephone lines the increase of capacity is remarkable. By use of VDSL modems the provided capacity is even higher - about $25 \mathrm{Mb} / \mathrm{s}$. Since each individual user 
seldom or never will take full advantage of this capacity, most of it will remain unused. There are two obvious reasons for this. The first is that the users do not have applications that continuously require that bandwidth. Secondly, users will normally subscribe to less bandwidth than the modem is able to provide, due to the operator's charging policies. The excess bandwidth is wasted in today's networks, and is therefore a loading capital cost for the network owners.

This paper describes an approach to make use of these unused resources to establish a real broadband mobile network based upon an evolvement of today's "privately owned" wireless LANs by extending the usability and supporting it by the fixed private access lines. It is assumed that wireless technology will become the winning technology for in-house communication both for residential and business users. The main idea is to open these networks for visitors that occasionally enter a "private" wireless LAN zone, and give them access to their service providers as if they were "at home". The visitors and the stationary users will share the capacity of wireless LANs and access lines according to a general service agreement between the involved parties.

Compared to conventional cellular mobile networks, which consist of a limited number of optimally located out-door base stations and antenna masts, the new network will consist of 'millions' of micro base stations randomly located in public as well as in private premises. Each of these micro base stations will be connected to the backbone broadband network over a casual type of access line. Hand-over protocols will enable seamless mobility across wireless LAN zones, possibly connected to different access providers, thus establishing a real broadband mobile network. Figure 1 below shows a functional illustration of the concept. The figure shows a mobile user moving through a number of wireless LAN zones connected to different access networks, while still maintaining his connections. A personal profile system ensures that the user's preferred services are maintained according to the QoS level each network can provide.

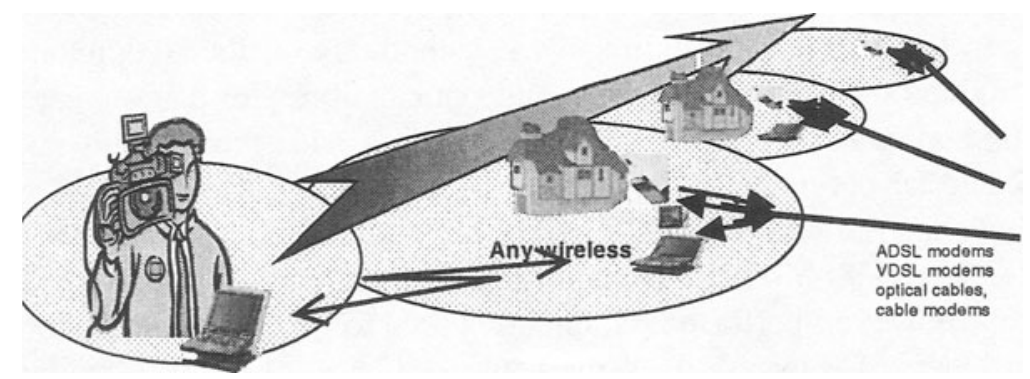

Figure 1. The Open Access Network scenario description 
The consecutive chapters of this paper contain a brief discussion of some of the most important features of the OAN. Chapter 2 contains a functional description of the total network and its components, while chapter 3 gives an evaluation of its performance. Chapter 4 elaborates on some aspects related to business incentives and models. The conclusive chapter 5 indicates how the OAN fits into an evolution path towards $3 \mathrm{G}$ and $4 \mathrm{G}$ mobile networks.

\section{FUNCTIONAL DESCRIPTION}

Figure 2 shows the general architecture and scenario description of an Open Access Network (OAN). From the left, a Moving User is traversing through two wireless Open Access Premises (OAP, i.e. home networks). The wireless Residential Gateways (wRG) are equipped with multi-protocol radio base stations (WLAN, HiperLAN, Bluetooth, etc.), enabling "any" wireless terminal to access the network. The OAPs are further on connected to the operator's access networks over any kind of broadband transmission medium, such as ADSL, VDSL, cable, or fibre. Each of the OAPs may be connected to different Network and Service Providers. Consequently, roaming agreements are required to enable service.

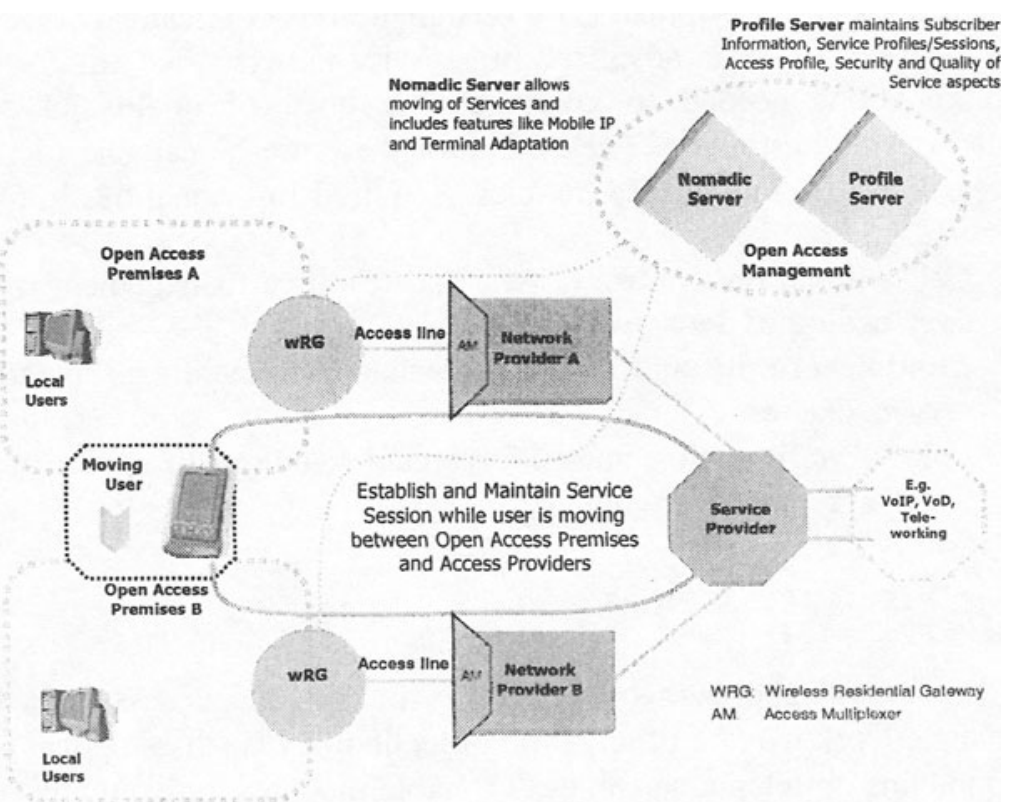

Figure 2. The Open Access Network architecture 
A mobile user of an OAN shall be able to connect and maintain his connections with his service providers while he is traversing the landscape from OAP to OAP. IP based mobility schemes as mobile IP may be used to maintain the connections while the user is moving between wRGs. The Nomadic Server controls the mobility features and includes terminal adaptions. The Profile Server contains and maintains the user's subscription information, service profiles/sessions, access profile, security, and QoS aspects. When the user enters a zone where his services cannot be maintained at the QoS level he is actually using, the services will be scaled down to a level that can be provided over the network of that zone. The QoS downgrade will also happen for instance if the user has to change from using a PC to using a PDA, which has other capabilities (e.g. another display format).

\subsection{Access Multiplexer (AM)}

The Access Multiplexer (AM) is the unit that terminates and concentrates the traffic from numerous access lines. In an access network based upon $\mathrm{xDSL}$ access lines, this unit is equivalent to the more commonly used term DSLAM (Digital Subscriber Line Access Multiplexer). For access networks based upon other technologies, the corresponding access units will have other names, like OLT (Optical Line Termination) for optical networks.

The AM located in an advanced full service network like an OAN must support functions needed to guarantee the level of quality of service requested by the customers. The AM must therefore be capable of sharing the access line based on agreed policies. Required functionalities in the AM will include:

- Call Admission Control (CAC) and resource management to avoid overloading of the network

- Multiple priority and queuing mechanisms enabling separation of service classes

- Policy configuration and enforcement handling to ensure that the usage agreements are not violated.

\subsection{Fixed access lines}

In the general approach to the OAN concept, the access lines to the backbone network may be of any type of technology, such as optical cables, xDSL modems on telephone cables, TV cable modems, or even any type of wireless solution as e.g. LMDS (Local Multipoint Distribution System). Among these alternatives, access over xDSL modems is probably the most relevant, since the telephone cable infrastructure covers nearly $100 \%$ of the 
population in many countries. Though it is commonly known that xDSL modems provide bandwidth in the range of megabits per second, it is worth mentioning that the technology is usually implemented asymmetrically. The highest bandwidth is in the downstream directions, while the upstream capacity is relatively limited, in the range of $0,5 \mathrm{Mb} / \mathrm{s}$ for ADSL and 2-4 $\mathrm{Mb} / \mathrm{s}$ for VDSL modems. This means that bandwidth may be a limited resource in an OAN, and resource management is therefore a critical issue.

\subsection{Wireless Residential Gateway (wRG)}

The wireless Residential Gateway performs the following functions:

- Terminating the access line with an appropriate transceiver, for instance an ADSL modem, to give access to the backbone network

- Multi-protocol Wireless LAN base station (WLAN, HiperLAN, Bluetooth, Ethernet, etc.), in order to allow "any" type of wireless and wireline terminal to connect to the network

- Priority, CAC (Connection Admission Control) mechanisms, and resource management of the wireless LAN to ensure fair traffic handling and prevent overload.

- Security and authentication mechanisms supporting VPNs and providing basic firewall and intrusion detection services. The wRGs should be able to establish VPNs between themselves, with other network elements, as well as with the user terminals.

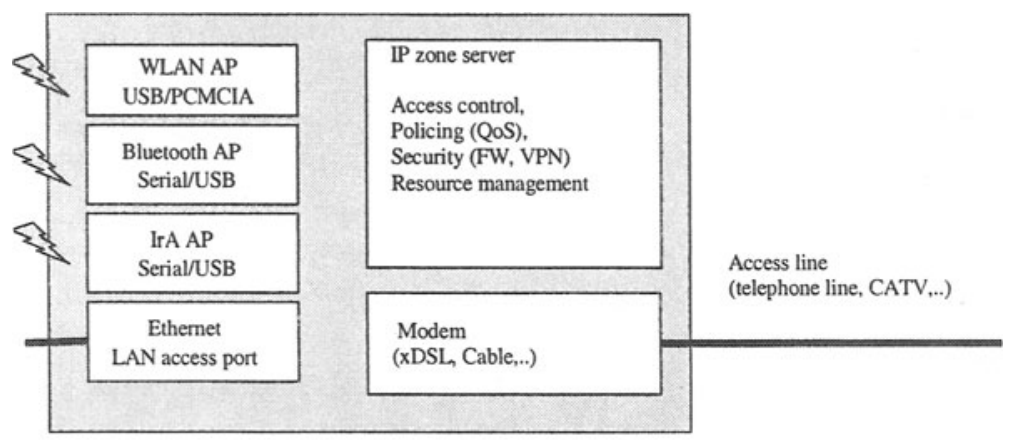

Figure 3. Wireless Residential Gateway

\section{$2.4 \quad$ Open Access Management}

The Nomadic and the Profile Servers perform the Open Access Management functions. These functions are located in the operator's domain on the network side of the AM. Each subscriber will be linked to a profile 
server and nomadic server in its home network similar to the home location register (HLR) in cellular networks like GSM.

The Nomadic Server keeps track of the location of the users that are traversing the networks e.g. by using Mobile IP to enable the necessary service mobility. The unit also realizes terminal adaptation in order to adapt the service to the terminal that currently is in use.

The Profile Server maintains the users' subscription information, security algorithms, and preferred service profiles/sessions. The server also contains the algorithms needed to adapt the services to the performance of actual networks. Hence, the Profile Server will have to collect data (bandwidth limitations, error rates, transmission delays, etc) from other network elements in order to optimise the quality of the offered service.

\subsection{Personal User Terminals (UT)}

The personal User Terminals (UT) may be any type of wireless or wireline terminals able to communicate over the diverse communication infrastructure supported by the wRG. The user terminal provides security mechanisms for authorization, authentication, access control, and data protection according to available encryption schemes. In addition, a security policy could enforce a minimal level of security involving virus detection and local firewalls.

\section{NETWORK PERFORMANCE}

The notation "network performance" covers a range of different properties of the network that influence how it performs towards the outside world. The term has different meanings depending on the observer's role in the network. The end-user has a different priority list of performance parameters, compared to the properties network operators may focus on. The end user will typically evaluate network performance according to the services available to him, the quality of them, and where he can receive them. The operator, on the other hand, will have a more global view and evaluate the performance based on strictly technical issues supporting a multitude of diverse users. The society, as a third entity, will also judge the network performance differently, probably focusing on the social benefits that the OAN concept may introduce.

This paper does not contain a complete evaluation of the network performance parameters, but rather a selection of the most critical ones. It is focused on issues that have relevance for this particular kind of network, i.e. on matters where it is expected that an Open Access Network will perform 
different from other networks, or where the OAN has different requirements than other networks. Each of the following sub-chapters contains an ingress explaining the reason for why this particular performance parameter has been chosen.

\subsection{Coverage estimation}

Compared to traditional strategies for implementing mobile networks, where dedicated equipment is used and antenna masts and base stations are optimally located to minimise investments, an OAN takes another approach. The OAN strategy is to use inexpensive equipment that is non-optimally located in residential homes, public and business premises, at the cost of an increased number of base stations. Since this is a completely new approach that has not been evaluated before, it is particularly interesting to look into.

To make coverage estimation simpler, it is assumed that a fixed broadband access network exists at the time of installation of the OAN. It is anticipated that most households and business users are connected to the backbone network with a mixture of ADSL/VDSL modems and optical fibres. It is further assumed that the new network is purely based upon the use of these existing resources i.e. that Micro Base Stations (MBS) are located in the users premises. The only external connection that can be used as a feeder line for the MBS is the user's access line. The concept does not take into account that additional base stations can be established elsewhere, using dedicated feeder lines in order to increase the radio coverage and make the covered areas unbroken.

According to public statistics in Norway about $75 \%$ of the population live in areas defined as urban settlements. An urban settlement is defined as an area where the distance between houses does not exceed 50 metres. This information can be used to give a rough estimate of the potential coverage for an OAN purely based upon MBSes located in the residential, business, and public premises.

Assuming that all households and business units are equipped with for instance WLAN networks (reach $<100$ metres) and that these are made public accessible, $100 \%$ coverage is achieved in urban areas since the distance between base stations is within acceptable margins for WLAN technology. This should not be understood as countrywide coverage, since the urban settlements only constitutes $0.7 \%$ of the total land area, leaving most of the country uncovered.

By providing the remaining part of the population with an appropriate wireless technology, coverage can be further increased. The rest of the population constitutes about 0.5 million households. Each base station covers an area of about $0,03 \mathrm{~km}^{2}$ and the total area covered is consequently 
$15,000 \mathrm{~km}^{2}$, i.e. $5 \%$ of the country's land area. The latter $5 \%$ does not constitute a continuously covered area, but rather an area with distributed "hot spots".

Using out-door antennas and other types of radio technology, the coverage zone per base station can be increased. A more detailed study will have to be done to estimate this potential.

\subsection{Bandwidth}

The OAN makes use of inexpensive wireless LAN technologies to realize the wireless part of a public mobile network. The base stations are not optimally located, thus one can expect crowded areas with too many base stations and other areas that are completely uncovered. Certain types of behaviour caused by the protocols used in such networks also influence the bandwidth performance. It is therefore of particular interest to evaluate how the network may perform with respect to the bandwidth available to users.

The bandwidth offered by the proposed OAN is limited by several conditions. The access line to the backbone network is one of them. An ADSL modem has an upstream bandwidth in the range of $0.5 \mathrm{Mb} / \mathrm{s}$, and is therefore a fundamental limitation. Using VDSL modems improves this, by increasing the upstream bandwidth to $2-4 \mathrm{Mb} / \mathrm{s}$. The downstream bandwidth is generally considered to be sufficient - in the range of $4 \mathrm{Mb} / \mathrm{s}$ for ADSL and $25 \mathrm{Mb} / \mathrm{s}$ for VDSL for high volume deployment. Cable modems provide capacity in the same range.

The second limiting condition is imposed by the chosen radio solution. Existing and up-coming standards for wireless LANs (HiperLAN/2 and IEEE $802.11 \mathrm{x}$ ). indicate net bandwidth in the range of $20-25 \mathrm{Mb} / \mathrm{s}$ over 30 metres. The bandwidth decreases rapidly over longer distances. At 50 metres the bandwidth falls to $10-15 \mathrm{Mb} / \mathrm{s}$ and at 100 metres the performance may be as low as $1-2 \mathrm{Mb} / \mathrm{s}$. Traffic load also degrades the performance of the wireless LAN. The higher the traffic is, the lower the throughput will be.

Although the above restrictions/limitations seem to disqualify the OAN, the performance is much higher than one should believe. In traditional cellular mobile networks, base stations are optimally located in order to cover as many users as possible, because of the high investment cost per base station. In the OAN, the investment per micro base station is anticipated to constitute only a fraction of the cellular mobile base stations, and the number of base stations can therefore be increased, each covering only a few potential users.

As an example of calculation, consider an area of $1 \mathrm{~km}^{2}$ with a population of 2000 families, each equipped with a micro base station fed over a) ADSL or b) VDSL. In case a) the total bandwidth distributed over 
the area is $8 \mathrm{~Gb} / \mathrm{s}$ downstream and $1 \mathrm{~Gb} / \mathrm{s}$ upstream. The similar figures for case b) are $50 \mathrm{~Gb} / \mathrm{s}$ and $8 \mathrm{~Gb} / \mathrm{s}$. Due to the granulated distribution of bandwidth, the OAN has nearly the same performance all over the area, while a traditional network performs worse at its borders.

\subsection{QoS}

Quality of Service is one of the most important performance parameters of any network, so also for an OAN. The inclusion of wireless LANs in a public mobile network is a new approach, and a careful evaluation is necessary. There are open issues both as regards QoS over wireless LANs, as well as over $\mathrm{xDSL}$ modem lines. Connecting wireless LANs and XDSL modem lines together further increases the need for studies.

Besides security, support for quality of service is one of the main issues for success in the future market. In the open access concept, as for all user communication, it is the perceived end-to-end QoS that must be ensured, either between two or more users or between a user and a network entity. Each segment of the communication link has to be provisioned with regards to QoS. In our context, these segments are the access link (e.g. ADSL, VDSL, and fibre optic links) and the wireless part at the user premises.

\subsubsection{QoS on the access line section}

The access link has to be able to support QoS for the different applications envisioned to be deployed by the clients. Since the access link is a shared resource with limited capacity, one has to be able to conduct service differentiation to ensure that the service requirements for certain applications are supported (e.g. real time applications). Proper queue handling (for instance based on Diffserv) is one potential solution for carrying out this service differentiation. In addition to the queue handling, proper resource management algorithms, possibly based on the user profiles, have to be in place so that the differentiation mechanisms may function properly.

Furthermore, there is a need for policy agreements both at the network side and at the home premises to prevent unauthorised access, as well as to prevent authorised users from flooding the link with data. To ensure that the traffic contracts are not violated, policy enforcements function need to be implemented. Data exceeding the agreed contracts may therefore be dropped or re-marked (ref DiffServ "marking"). 


\subsubsection{QoS on the wireless section}

QoS also has to be ensured across the wireless section. Most of the current WLAN products do not support QoS. However, new up-coming standards from IEEE and ETSI define functionalities that will meet the QoS requirements demanded from the future broadband communication infrastructure. The main difference between the IEEE and the ETSI recommendations comes from their different backgrounds. While the IEEE 802.11 recommendations are based on IP/Ethernet thinking, the ETSI protocol HiperLAN is brought forward from an ATM background, which supports strict QoS requirements. Bluetooth, as a personal wireless network, implements connection-less and connection-oriented channels, suitable for handling real time applications as voice as well as data applications. Nevertheless, regardless of which wireless technology is deployed, the QoS requirement has to be met, and it would be the operators responsibility to ensure that the networks are properly scaled for the estimated number of users in a region taking into account the error rates due to weather conditions, interference, cross talk etc.

Since the open access concept allows nomadic users to deploy the private access lines, a resource uncertainty is introduced into a communication session. If there are not enough available resources in the visited LAN there is a possibility that the QoS requirements cannot be met. This is due to the uncertainty in number of simultaneous users on the network and/or because the distance to the actual base station is too great. The uncertainty is further increased due to the problem of estimating the available bandwidth on the LAN, since the bandwidth decreases as a function of the number of users and the traffic generated by each of them. An efficient way of solving these problems will have to be studied.

\subsection{Security and authentication issues}

An OAN requires a strong, but flexible security architecture, as users will be sharing access lines, wireless LANs, as well as other resources. A user must be authenticated to the system, and mechanisms for confidentiality and access control are key elements.

There are many available complementary security systems, and security and authentication can be implemented in different ways. Today, secure access to services from an open network is usually implemented using secure protocols (like ssh and SSL) or VPN (with IPSec, SSL, etc.). A protocol for wireless encryption has been developed for the IEEE802.11 protocol, but this protocol is fundamentally flawed. 
VPN provides an authenticated, encrypted connection between hosts, and it is suitable for securing the connection between the wRGs and between the wRGs and the fixed network. However, the VPN solution is connectionbased and processing intensive, and it is currently not suitable for implementing seamless mobility for all user clients. The problem in such a setting is that the encrypted channel has to be renegotiated at every handover. This is a time-consuming job that may prohibit real-time applications and some TCP-connections. The problem of renegotiation may be addressed by wrapping Mobile IP around IPSEC. However, implementations that wrap IPSEC around Mobile IP do not solve this problem. Furthermore, a VPN is dependent on platform security, as it does not provide end-to-end security at the user or application level. A VPN only protects the communication at the network layer.

In addition to communication security, it is necessary to implement a security policy that preserves overall system security. The wireless residential gateway should provide firewall and intrusion detection services, while the client security policy should define a minimum-security level using local firewalls, intrusion detection, and virus detection.

An OAN should support several modes of authentication, including strong authentication based on digital certificates or biometric methods. The scheme also has to be flexible in the sense that different users have different rights or capabilities according to what services they subscribe to. Subscribers of specific services have to be authenticated on a single sign basis and given access to resources and services available to them.

An Object Security scheme based on PKI (Public Key Infrastructure) could counter some of the limitations of the VPN. In such a scheme, digitally signed data objects can be stored and transmitted in encrypted form. Data objects, such as for instance user profile information, calendars, and contact lists can be retrieved and decrypted by a user at any client. This provides end-to-end security at the application level from the creator of the object to the end user. The idea behind Object Security is that the integrity and confidentiality of user data should depend on protection of data objects themselves, and not on platform or communication security alone. Also, the objects can be mobile, in the sense that they are available in a transparent manner on the OAN. 


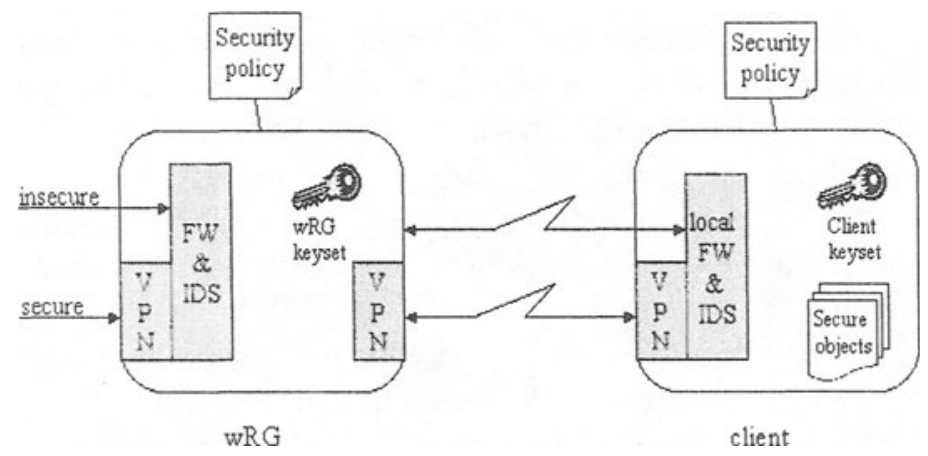

Figure 4. OAN security architecture

Figure 4 shows a security architecture that supports both secure VPNconnections as well as insecure connections. The security is divided in two levels, so that both the wRG and the client implement firewall and intrusion detection services. The figure shows that both the wRG and the client can authenticate using their own key set. The client has downloaded a number of secure objects, containing for instance profile information, contact lists, calendars, and so on. In addition, the figure shows the security policies that define and enforce minimal security levels for the wRGs and the clients.

\subsection{Mobility}

An OAN requires seamless mobility between different wireless residential gateways and between different access technologies. Both the wireless residential gateways and the user clients have to support session level mobility, so that a user can resume any session through another gateway or user terminal.

The OAN assumes that users have open access to wireless residential gateways, regardless of their current location and their network solutions at home. Residential areas are covered with wireless access technologies providing access to the backbone over residential fixed lines. A user traveling through such an area is offered continuous connectivity in the same manner as in the existing GSM network.

Mobile-IP can be used to provide mobility between home and the public place, but the current protocol has limitation with regards to QoS handling, real-time performance, and security. These limitations will have to be addressed in an OAN. In addition, the OAN depends on support for handover features between the different access technologies (for instance WLAN and Bluetooth) in order to provide seamless mobility. 


\section{BUSINESS ASPECTS}

The open access concept will give rise to new business opportunities. The concept involves both an optimisation component of the broadband access lines and the aspects of wireless broadband WLAN coverage, including mobility and new service deployment. Of course the realisation of the network described above may meet many obstacles because of its radical new approach to utilise the privately owned network resources. The home access networks are today considered to be under full private ownership, thus leaving the responsibility of acquisition and maintenance to the owners. The same is partly true for the access line, which is generally owned by a network provider, although the line itself is dedicated to each individual user. Sharing these resources with others will consequently be perceived as a reduction of existing exclusive rights. The success of the OAN depends on sufficient incentives for the involved parties to be willing to adopt the concept. The private user, the visited users that are roaming in the area, the network provider, as well as service providers all play a role in the business case to various degrees.

\subsection{Ownership of the wRG}

The ownership of the wRG is a crucial question. It may be owned and operated either by the network operator, the resident users, or provided by a third party.

Up until now the operator has not been involved in the network infrastructure inside the private sphere neither within ordinary housing or business facilities. If the user owns and operates the wRG itself, there is no big difference from the infrastructure of today, except for the fact that the access line is shared with other users completely on the basis of the resident users own will. The user would configure the wRG suited only to its own personal needs. It is not certain that everyone would be willing to install and support WLAN coverage for inbound roamers, and the benefits of full outdoor coverage would be jeopardised. Even if the user owns the wRG, the operator has to configure the AM to handle different user identities towards each access line with the following resource and usage priority functionality. The functionality of the wRG and the AM are closely linked. The user would be in control of how the access line is used, how the policy of usage is configured, how to conduct the authorisation etc. This may require quite some work, and the question remains whether the user is interested in taking this responsibility.

If the operators owns the equipment and handles all configuration, maintenance, upgrade, etc., a burden is taken off the shoulders of the users. 
On the other hand, the operator then enters the private sphere of the user. The operator could in a better way make sure that the community supports full WLAN coverage for inbound roamers, and thereby support service continuity while moving within the housing or business facilities. This would be an asset to all users living or staying in the area. It would be a collective benefit.

For the user it would make minor difference if the wRG is owned and operated by a third party. It could however make a difference to the network operator since the third party doesn't necessarily have the same incentives for launching the services and upgrades as a network provider, which would try to increase the usage of the network in terms of increased traffic volume.

There are laws that restrict intrusion into the private sphere. If someone restricts himself or herself from letting operators install equipment in their premises they have a right to do so. Installation of $w R G$ s has to be conducted on a voluntarily basis. It all comes down to how the user perceives the benefits of deploying the OAN. Firstly, the interests of installing it in the first place, secondly the choice of owning it itself or letting the operator own it and take responsibility for configuration, conducting resource sharing of the access line, configuration of the policy and QoS functionality, as well as the charging mechanisms.

In the following, only the business aspects of the case where an operator owns and operates the wRG is conducted. If the user or a third party owns the wRG, other business aspects might be relevant. The business aspects focus on the user and network operator. Only some minor considerations are given regarding the service providers.

\subsection{User benefits and incentives}

A user may act in different roles. In one moment a user acts as a stationary user residing inside a personal sphere. In this context he can access all his private wireless devises through a unified access where all devises may intercommunicate and exchange information. The personal sphere is fully configured, maintained and upgraded by an external party, being either the network operator or a service provider. The user is not worried about the communication infrastructure, which will be quite similar to a public wireless network like GSM or UMTS. Utilising a well known authentication method, the user can exchange context, move around outside the private premises, and still be attached to both his own private devices as well as to the internet and public backbone networks and services. The wireless device using different kinds of WLAN standards works just as well indoors, as when out walking or visiting a neighbour. Changing context again, the user may roam to another area and become a "visitor" in the new 
area. The concept works just as good. The user uses the same authentication method, stored in the profile register in the home network. He may access the backbone and his own private sphere by utilizing the access lines of one of the private residents. The local router routes the session to his home wireless access gateway. The user will experience an increase in service provisioning by always being able to reach the private sphere (home server, alarm-cameras, etc.), as well as being connected to a broadband wireless network with an increased service portfolio as compared to GSM and the upcoming UMTS network. A natural way of giving incentives to the stationary users is to offer them economical benefits, for instance by repaying them a share of the traffic generated from 'their' local areas. Different models for economical incentives for the user to deploy the concept are possible.

Making the stationary end-user's (the host's) access lines and wireless home networks open for public use impacts the user in several ways. First of all he will have to share the access resources with others. As long as there are enough resources he will be unaware of the resource sharing. In some cases, however, the visitor and the host will try to access the same resources. Priority schemes are one way to decide in the conflict. It is not always given that the host shall have the highest priority for any service he may try to initiate. The visitor may have higher priority, either by the nature of the actual service (for instance a real time service), or because he is willing to pay more for a given service. The host may also feel that he opens his private sphere for the public, and he must be convinced that his privacy and security requirements are protected in appropriate ways.

\subsection{Benefits and incentives for network owners}

For the network provider potential revenue may be gathered from various sources, like broadband access, owning and supporting the wireless residential gateway, traffic increase from roaming customers and visiting residents accessing the access lines, as well as traffic increase due to new service deployment. The access lines will act as the connecting network between the wireless LAN base stations, whereby the network operator in fact offers a mobile broadband network based upon planned and existing network resources. By installing wireless residential gateways in the private sphere, the customers are offered a local area network for their own use as well as the possibility to share it with roaming mobile users entering the coverage areas. Huge new investment costs may be avoided, since the network operators already have invested a profound amount of money in the existing access lines and planned upgrade of the access networks. 
Today, optical cables are dug down in the ground, and XDSL modems are installed to provide the stationary users with more bandwidth. It would be a huge benefit for the operators to be able to utilise this capacity by including WLAN access and roaming users access. This would mean a broader service portfolio and thereby traffic increase on the existing access network infrastructure. We have already seen a shift in the direction where the national cellular wireless networks are becoming an increasingly important carrier for voice traffic. In the future we may envision that all voice traffic will be based on wireless systems. Integrating mobile services and being able to invite mobile WLAN users to deploy the same access network may increase the income potential for the network owner even if the voice traffic is taken by other wireless systems. If WLAN may support voice and other real time applications, this voice capturing may be hindered to a certain degree.

A less obvious issue is the potential revenue from the equipment itself and the new business model of the operator owning and operating the wireless residential gateway. How much is the customer willing to pay to be able to put the burden of configuring, maintaining and upgrading the gateway and the WLAN coverage onto the operator? Today, it is difficult to give an answer to this, since the concept is so new and radical. Eventually, when the customer gets more used to sharing content and letting "somebody else do the dirty job", the business model may become more accepted, and it may become a potential revenue driving concept.

For the network operators one of the most important issues is to get traffic onto the network. The evolving peer-to-peer networking, where traffic is exchanged outside the operators charging domain, may be looked upon as a threat to network operators today. Since it is quite obvious that peer-topeer computing will emerge quite extensively, the issue would be how to best take advantage of this kind of communication to increase the traffic across the access and into the operators charging domain. The open access concept may be one way to try to benefit from the P2P communication since the operators charging sphere is pushed further towards the customers.

\subsection{Benefits and incentives for service providers}

The open access concept will also be a new business area for service providers, complementary to GSM, GPRS, and UMTS. New mobile services specially suited for broadband mobile network may be launched in the same packet as delivered to the household today. In contrary to the limited bandwidth of GSM and UMTS, the same services may be used on stationary and mobile devices when deploying broadband wireless networks. 


\section{CONCLUSION}

The open access architecture can be seen as a step towards the $3 \mathrm{G}$ and $4 \mathrm{G}$ mobile networks. The $3 \mathrm{G}$ mobile network is intended to provide access rates in the range of $1-2 \mathrm{Mb} / \mathrm{s}$, while the $4 \mathrm{G}$ mobile network pushes the access rates further up to $20 \mathrm{Mb} / \mathrm{s}$ and unifies the different wireless networks like the global coverage public cellular networks (GSM/UMTS), the hot spot wireless LAN networks (IEEE 802.11, HiperLAN/2), as well as personal area networks such as Bluetooth.

With an OAN approach as described in this paper, we may envision seamless integration between different personal wireless technologies, private and public WLANs, and public cellular systems like GSM and UMTS. With a limited number of devices, the users may utilise a vast variety of services both from within his own personal sphere and when roaming around in public areas nationally and internationally. The slogans of "make it easy" and "always connected" together with personalisation is in the same footprint as the concept of the open access. With a unique security keyset, different user profiles, and various wireless technologies on the device, the user is able to roam around and always be able to access the personal sphere and public backbone.

The OAN architecture solves the problem of rolling out cost-efficient infrastructures for future $3 \mathrm{G}$ and $4 \mathrm{G}$ mobile networks. Over the next few years, optical cable and millions of ADSL and VDSL modems will be installed in the fixed network, thus huge bandwidth is distributed far out in the telephone access network. Using these broadband access lines as feeder links for millions of wRGs, the potential bandwidth derivable by the users is in line with the above $4 \mathrm{G}$ expectations.

The existing telephone access networks have near $100 \%$ coverage in industrialised countries. The OAN concept therefore has the potential to reach $100 \%$ coverage in urban areas and spot-wise coverage in the less populated areas. This is an important quality of the OAN that will impact on future decisions towards the target network.

\section{REFERENCES}

- ETSI Hiperlan specifications, http://www.etsi.org/t_news/0005_bran.htm

- Mobile IP, the internet unplugged, J. D. Solomon, Prentice Hall, 1997

- Weaknesses in the Key Scheduling Algorithm for RC4, Scott Fluhrer, Itsik Mantin, and Adi Shamir, Selected Areas in Cryptography 2000 